\title{
Infratentorial Tuberculoma Mimics Tumor in Immunocompetent Adults: An Analysis of 11 Patients*
}

\author{
Ning Guo', Fan Huang ${ }^{2}$, Xi Chen ${ }^{3}$, Minying Zheng ${ }^{2}$, Dexia Zhong ${ }^{2}$, Yanqing Feng ${ }^{2 \#}$ \\ ${ }^{1}$ Department of Neurosurgery, The First Affiliated Hospital of Sun Yat-sen University, Guangzhou, China \\ ${ }^{2}$ Department of Neurology, Guangdong Key Laboratory for Diagnosis and Treatment of Major Neurological Diseases, \\ National Key Clinical Department, and National Key Discipline, The First Affiliated Hospital of Sun Yat-sen University, \\ Guangzhou, China \\ ${ }^{3}$ Department of Rehabilitation Medicine, The First Affiliated Hospital of Sun Yat-sen University, Guangzhou, China \\ Email: "fyqgz@sina.com
}

Received April 20, 2013; revised May 19, 2013; accepted June 3, 2013

Copyright (C) 2013 Ning Guo et al. This is an open access article distributed under the Creative Commons Attribution License, which permits unrestricted use, distribution, and reproduction in any medium, provided the original work is properly cited.

\begin{abstract}
Backgrounds: Tuberculoma is a granulomatous inflammatory process mimicking a neoplasm, both clinically and radiologically. Although those with an infratentorial origin are rare, this disease is still a diagnostic challenge using conventional workup. However, this disease should not be overlooked because it is essentially curable with proper diagnosis and therapy, usually, a Mycobacterium Tuberculosis (MTB) DNA test is performed. Methods: We retrospectively analyzed the clinical presentations, CSF results, and images of 11 MTB DNA positive and clinically cured cases of infratentorial tuberculoma. Results: Infratentorial tuberculoma usually deteriorated before antituberculosis treatment (ATT). Magnetic resonance imaging showed space-occupying lesions without specific features, 4 within the cerebellum and 7 within the brainstem. Evidence of systemic tuberculosis was found in only 1 case. Clinical manifestations included various combinations of focal signs and symptoms in the brain stem and cerebellum. Cerebrospinal fluid (CSF) findings were also nonspecific. The diagnoses of these cases were based on the positive tests of a nested polymerase chain reaction (N-PCR) assay. Trial therapy with antituberculous drugs resulted in clinical improvement, as documented by MRI in all patients. Conclusions: Infratentorial tuberculoma should be suspected in patients with infratentorial space-occupying lesions who live in geographic areas where tuberculosis is endemic.
\end{abstract}

Keywords: Infratentorial; Tuberculoma; Tumor; Diagnosis

\section{Introduction}

Tuberculosis involvement of the central nervous system continues to represent a serious problem, particularly in developing countries [1]. Parenchymal tuberculosis is more common in human immunodeficiency virus (HIV)infected patients and can occur with or without meningitis [2]. However, it is an uncommon entity especially in the immunocompetent adult. Tuberculomas are granulomas of tuberculous origin which behaves as space-occupying lesions, and comprise 5\% - 30\% of all intracranial masses [3]. Tuberculomas may occur anywhere in the central nervous system, although in the brain they tend to be supratentorial in adults and infratentorial in children [4]. Although potentially curable, tuberculomas often present a diagnostic puzzle, and the diagnosis may

\footnotetext{
"Conflict of interest: The authors declare that they have no competing financial interests.

${ }^{\#}$ Corresponding author.
}

not be considered early in a patient's course.

Infratentorial tuberculoma is life-threatening, and clinical manifestations included various combinations of focal signs and symptoms of subacute onset, similar to those produced by other space-occupying lesions in the brain stem and cerebellum. There are isolated adults cases with brainstem and cerebellar tuberculomas reported worldwide in the last 10 years [5-9], wherein diagnosis was mainly at the time of surgery or at necropsy. The clinical diagnosis of this condition is difficult in practice, and a delay in the diagnosis of this curable disease contributes to significant neurologic sequelae. Conventional microbiology has limited utility, whereas efficient diagnosis by detection of Mycobacterium tuberculosis (MTB) DNA can provide a rapid diagnosis of CNS TB, possibly saving lives. We herein present eleven patients with infratentorial tuberculoma, diagnoses were established on positive test of MTB DNA. The purpose 
of this study is to stress that infratentorial tuberculoma may be misdiagnosed as a tumor.

\section{Patients and Methods}

This retrospective study was carried out at the Neurology Department of the First Affiliated Hospital of Sun Yat-sen University in southern China between 2005 and 2012. Our retrospective study consisted of 11 patients whose infratentorial space-occupying lesions were considered to be tumors, but were diagnosed as tuberculomas by MPB 64 targeted nested PCR. The inclusion criteria were: 1) space-occupying lesions in the brain stem and/or cerebellum on magnetic resonance imaging (MRI); 2) positive CSF MPB 64-targeted nested PCR in our lab; 3) good response to antituberculosis treatment.

The university ethics committee approved the study. Written informed consent was obtained from patients who agreed to participate in the study.

\section{Results}

\subsection{Baseline Characteristics of Study Patients}

The age of the patients ranged from 17 to 75 years (mean 44.2 years). Three were female and eight patients were male (F/M ratio: 37.5\%). The mean duration of neurological symptoms prior to admission was 51 days (14 days to 5 months). Double vision (4 cases), dizziness (4), unsteady gait (3), slurred speech (2), numbness and weakness on one side of the face (2), headache (2), hemiparesia (2), seizures (1), and vomiting (1). The evidence of tuberculosis in the lung was found in 1 patient. Fever occurred in just one patient. Two patients had a prior history of tuberculosis.

\subsection{Cerebrospinal Fluid Study}

The CSF findings on admission were as follows: elevated opening pressure $\left(>200 \mathrm{~mm} \mathrm{H}_{2} \mathrm{O}\right)$ was present in 1 patient, CSF protein levels were increased $(>0.45 \mathrm{~g} / \mathrm{L})$ in 6 patients, 4 patients had elevated CSF leukocyte counts, and 1 patient revealed a low CSF chloride level. The $\mathrm{CSF} /$ serum glucose ratio was normal for all patients. Overall, CSF was abnormal in 8 of 11 patients. CSF microscopy for AFB and CSF cultures were all negative. N-PCR for MTB DNA was positive in repeated tests for all these patients.

\subsection{Imaging Findings}

Magnetic resonance imaging (MRI) findings in all patients showed infratentorial space-occupying lesions: an iso-hipo intense in $\mathrm{T} 1$ sequence, a contrast-enhanced lesion in $\mathrm{T} 1$ sequence and hyper intense lesion in T2 sequence. Radiologically, those lesions were considered to be tumors. Solitary lesions were more prevalent. Among the lesions, four were located in the cerebellum (4/11) (Figure 1), seven was intramedullary (7/11), whereas only 1 had brainstem contrast-enhancing lesions and right basilar involvement (Figure 2). Hydrocephalus was observed in 1 patient (Figure 1).

\subsection{Treatment Efficacy and Follow-Up}

All patients experienced good response in the early course of ATT treatment. Paradoxical responses were seen in 2 patients. The No 1 patient experienced worsen

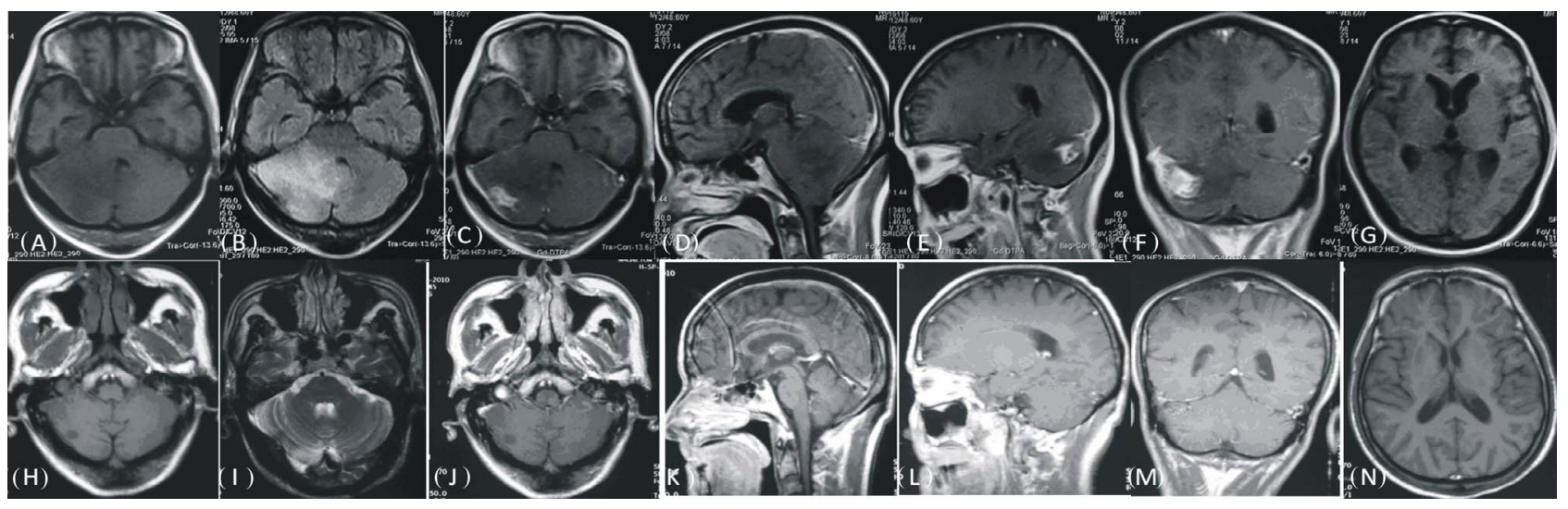

Figure 1. MRI changes before and after treatment. (A): MRI at presentation on axial T1-weighted image showing mild diffuse low signal intensitiy area in the whole right cerebellar hemisphere, extending into the left side. (B): axial T2-weighted image disclosing T2 high signal abnormality extending from the pons into the proximal right cerebellar peduncle and throughout the whole right cerebellar hemisphere. (C): Contrast-enhanced axial T1 showing right cerebellar tuberculoma with irregular enhancement and perifocal oedema. (D): cerebellum appears hypointence on sagittal T1W image. (E,F): contrast-enhanced lesion and perifocal oedema was present. (G): Mild hydrocephalus was observed in axial T1 image. (H): T1-weighted image after 2 years treatment, (I): axial T2-weighted image reveals the hyperintensity lesion resolved following 2 year of ATT. (J): Contrast-enhanced axial T1 is normal. (K): Cerebellum on sagittal T1W image. (L,M): contrast-enhanced lesion and perifocal oedema was absent. $(\mathrm{N})$ : mild hydrocephalus resolved on axial T2 weighted image. 


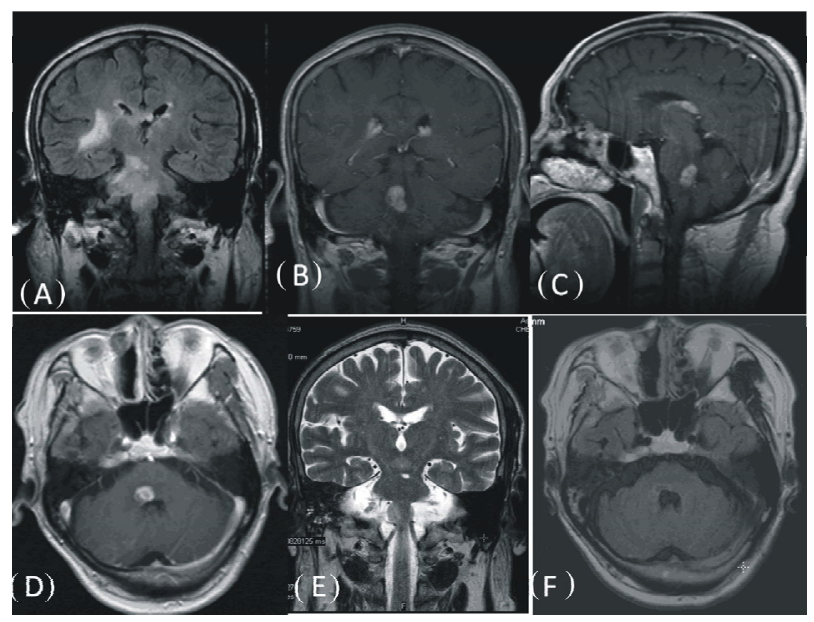

Figure 2. Changes of lesion depicted by MRI. (A): FLAIR of brain MRI revealed extensive high signal lesions in the brain stem and right basal ganglia region, (B) (C) (D) mass lesion was present on coronal, sagittal and axial contrast-enhanced T1-weighted images. (E): Repeat MRI performed 1 year later after the initiation of ATT, showing lesions were absent on coronal T2-weighted image. (F): lesion disappeared on axial FLAIR sequences.

ing headaches with increased open CSF pressure during the second weeks of therapy. The headaches resolved in 12 days after mannitol treatment. The No. 11 patient was seen worsening dizziness with paradoxical enlargement of mass lesions, and it lasted 10 days. No alteration was made in the drug regimen in this patient.

All 11 patients were followed up regularly at the outpatient clinic. Side effects due to antituberculosis therapy developed in 3 patients. Mild gastrointestinal irritation was seen in 2 patients, and mild elevation in hepatic enzymes developed in 1 . All these side effects were resolved quickly by active therapy and were able to continue the ATT treatment. The mean follow-up period was 4 years (range: 28 months to 6 years). Permanent neurological sequelae were observed in 1 patient. Two-year observations based on MRI imaging were completed for all patients. All infratentorial lesions were resolved after ATT treatment. No recurrent lesions were observed in the follow up time.

\subsection{Case Reports}

Case 1: A 59-year-old previously healthy male was referred to us with an occipital headache that he had for fifty days. He complained of gait disorder, throbbing headaches, and vomiting with inability to walk for 7 days. T1-weighted MR imaging revealed a low signal intensity area in the right cerebellar hemisphere and vermis (Figure 1(A)). Fluid-attenuated inversion recovery (FLAIR) disclosed an extensive high signal lesion extending into the left hemisphere, with brainstem compression (Figure 1(B)). Contrast-enhanced T1WI showed irregular en- hanced lesions with mass effect (Figures 1(C)-(F)). Mild obstructive hydrocephalus was also observed (Figure 1(G)). There was no other clinical evidence of a tuberculous process. On admission, results of routine laboratory tests and chest X-ray were normal. Spinal tap showed elevated CSF pressure $\left(290 \mathrm{~mm} \mathrm{H}_{2} \mathrm{O}\right)$, and the following: protein $1020 \mathrm{mg} / \mathrm{L}$, glucose of $4.2 \mathrm{mmol} / \mathrm{L}$, and WBC $2 / \mathrm{mm}^{3}$. The CSF culture was negative for Mycobacterium tuberculosis. However, MPB64-targeted N-PCR was positive. Tuberculoma was therefore considered. He was given an ATT regimen consisting of isoniazid (600 mg/day), rifampin (600 mg/day), pyrazinamide (1500 mg/day), and amikacin (600 mg/day), and together with mannitol (125 ml q8h). His headache quickly decreased. However, after 10 days of treatment, his headache recurred with vomiting. Another spinal tap revealed a CSF pressure of $350 \mathrm{~mm} \mathrm{H}_{2} \mathrm{O}$, and CSF: protein at $693.6 \mathrm{mg} / \mathrm{L}$, glucose at $3.7 \mathrm{mmol} / \mathrm{L}$, and $\mathrm{WBC}$ at $8 / \mathrm{mm}^{3}$. Mannitol was increased to $250 \mathrm{ml}$ iv drip q8h. After 12 days of active treatment, his headache decreased greatly. One month after admission, the third spinal tap showed CSF pressure decreased to $175 \mathrm{~mm} \mathrm{H}_{2} \mathrm{O}$. CSF: protein $416.1 \mathrm{mg} / \mathrm{L}$, glucose at $3.1 \mathrm{mmol} / \mathrm{L}$, and WBC at $0 / \mathrm{mm}^{3}$. The patient continued an ATT regimen for 24 months. A brain MRI obtained 24 months after ATT revealed the mass lesion is absent (Figures $\mathbf{1}(\mathbf{H})-(\mathbf{N})$ ).

Case 2: In June 2005, a 69-year-old Chinese man presented with diplopia and right face weakness and atrophy that lasted for 5 months, and right-sided weakness, hyperesthesia and slurred speech for that lasted for two weeks. He did not present with any cough, hemoptysis, fevers, chills, or weight loss. On neurological examination, he was alert and well oriented and did not have neck stiffness or other signs of meningeal irritation. He was found to have bilateral gaze-evoked nystagmus (with a rotatory component), and a right sixth nerve palsy with hyperreflexia and hyperesthesia on the left side of the body. There was decreased sensation over the right $\mathrm{V} 1$ 3 dermatomes, and the right corneal reflex and gag reflex were absent. There was a mild facial palsy and taste was impaired on the right side. A hemogram showed an elevated erythrocyte sedimentation rate of $16 \mathrm{~mm} / \mathrm{h}$ and a normal white blood count. The Mantoux test was positive. A chest X-ray revealed right apical parenchymal infiltrates and consolidation. Sputum specimens stained negative for Acid Fast Bacilllus (AFB). FLAIR of brain MRI revealed extensive high signal lesions in the brain stem and right basal ganglia region (Figure 2(A)). The post contrast T1 images showed a single lobulated enhanced mass within the brain stem (Figures 2(B)-(D)). A diagnosis of CNS lymphoma was considered and this was supported by whole-body FDG PET/CT that showed multiple hypermetabolic nodular lesions throughout the neck, chest, abdomen, and pelvis. CSF examination re- 
vealed a total protein level of $780 \mathrm{mg} / \mathrm{L}$, a glucose level of $2.8 \mathrm{mmol} / \mathrm{L}$, and 3-lymphocytes/L. The CSF culture was negative for MTB. However, MPB 64-targeted NPCR was positive. A neck lymph node biopsy revealed a chronic infection. On the basis of these findings, a presumptive diagnosis of brainstem tuberculoma was made, and anti-tuberculous therapy was started. The patient improved quickly. A follow-up MRI after 1 year of treatment showed that the lesions had disappeared. The patient completed 2-year of antituberculosis treatment. At a 6-year follow-up, he demonstrated a good recovery, although he still complained of mild diplopia.

\section{Discussion}

The aim of this study was to increase the awareness of infratentorial tuberculoma, especially in patients with infratentorial space-occupying lesions who live in geographic areas where tuberculosis is endemic. Despite being preventable and curable, this disease is still a diagnostic challenge using conventional workup. A definitive diagnosis could not be made using routine CSF tests, and conventional MR because similar MR findings are more commonly found in infratentorial tumors.

Infratentorial tuberculoma poses a more risk to the patient life than supratentorial one, and are seldom reported. It may present with isolated mass lesion without evidence of systemic tuberculosis, mimicking tumors rather than the infectious process [11]. As the cases in this study, clinical manifestations depend largely on their location, systemic TB is not common, and chest X-ray was normal in all except one patient who had lung tuberculosis. The Tuberculin skin test, ESR and other conventional testing techniques were not helpful in suggesting an infectious cause. In this case series, only one patient is associated with concomitant meningitis; this is similar with the findings by other authors [12,13], indicating CNS tuberculomas are less accompanied by evidence of coexistant meningeal involvement.

Unfortunately, diagnosis of CNS TB remains difficult. Although those reports demonstrated that neuroradiological findings might be helpful in the diagnosis of tuberculosis, However, MRI of the brain cannot distinguish between gliomas, lymphomas and tuberculomas with complete accuracy. Its diagnosis usually necessitates preferably, a stereotactic brain biopsy followed by, a histopathological examination. However, this invasive diagnostic procedure cannot identify etiologic agents in all patients [14]. Bayindir [15] reported 23 pathologically proven cases, there are only 4 cases demonstrated acidfast bacilli. There are also significant risks [16] about this technique, especially in infratentorial lesions. Talamas [17] reported a brainstem tuberculoma patient from Mexico in 1989, which had a surgical biopsy, and died
$36 \mathrm{~h}$ after, highlighting the dangers of brainstem biopsy.

Today, with the increasing incidence of tuberculosis, various studies have demonstrated that early diagnosis and treatment are essential since the patient outcome of CNS tuberculosis depends on the clinical stage and on the age of the patient [18]. A correct diagnosis and early institution of specific treatment is warranted. PCR-based tests have been employed in the diagnosis of CNS TB with reasonable success [18]. However, their diagnostic efficacy are usually be questioned by their specificity for distinguishing CNS TB from other CNS disease. Several other CNS diseases $[10,20]$ had been found to be associated with mycobacteria infection over the past 3 years. Therefore neurologist should be aware of the limitations to the notion of "sensitivity and specificity” about MTB PCR tests. The possibilities exists that mycobacteria infection might act as a potential contributing or pathogenetic factor in some certain CNS diseases because of their unknown etiologies. However, our study employed the nested-PCR test in the detection of MTB DNA, in large part because of the frequent difficulty in detecting tubercle bacilli in smears or CSF cultures.

In our case series, one patient presented with a "paradoxical” enlargement of a lesion during early treatment, and another patient presented with transient worsening due to intracranial pressure. This is presumably an immunologic response and usually a transient phenomenon [21]. Some cases may require intensive monitoring and treatment of raised intracranial pressure resulting from the expansion of the mass. Some patients have shown sudden deterioration in neurologic status, and even died after initiation of antituberculous therapy in literature [22].

There are methodological limitations to the current study. This is a small numbered retrospective study. The study did not evaluate the specificity and sensitivity of the nested-PCR test in the detection of MTB DNA in $\mathrm{CSF}$. And therefore it is difficult to judge the true value of the test. However, It can be alert to us that tuberculoma may be misdiagnosed to be a tumor. For this reason, clinicians must always be aware of it and consider it in the differential diagnosis.

\section{Conclusion}

Our limited clinical experience of these disorders indicates that infratentorial tuberculomas should be suspected in patients with focal space-occupying lesions who live in geographic areas where tuberculosis is endemic. Diagnosis can be made by the combination of clinical presentation, imaging, MTB DNA test and response to treatment.

\section{Acknowledgements}

This work was supported in part by Guangdong Medical 
Research Foundation (Grant No. 2012B03180067).

\section{REFERENCES}

[1] A. Cherian and S. V. Thomas, "Central Nervous System Tuberculosis,” African Health Sciences, Vol. 11, No. 1, 2011, pp. 116-127.

[2] P. F. Barnes, A. B. Bloch, P. T. Davidson and D. E. Snider Jr., "Tuberculosis in Patients with Human Immunodeficiency Virus Infection,” The New England Journal of Medicine, Vol. 324, No. 23, 1991, pp. 1644-1650. doi:10.1056/NEJM199106063242307

[3] M. R. Gropper, M. Schulder, H. L. Duran and L. Wolansky, "Cerebral Tuberculosis with Expansion into Brainstem Tuberculoma," Journal of Neurosurgery, Vol. 81, No. 6, 1994, pp. 927-931. doi:10.3171/jns.1994.81.6.0927

[4] H. M. Dastur and A. D. Desai, "A Comparative Study of Brain Tuberculomas and Gliomas Based on 107 Case Records of Each,” Brain, Vol. 88, No. 2, 1965, pp. 375396. doi:10.1093/brain/88.2.375

[5] S. I. Poonnoose, S. Singh and V. Rajshekhar, "Giant Cerebellar Tuberculoma Mimicking a Malignant Tumor,” Neuroradiology, Vol. 46, No. 2, 2004, pp. 136-139. doi:10.1007/s00234-003-1148-0

[6] S. Sathyanarayana, M. K. Baskaya, M. Fowler, R. Roberts and A. Nanda, "Solitary Tuberculoma of the Cerebellopontine Angle: A Rare Presentation,” Journal of Clinical Neuroscience, Vol. 10, No. 1, 2003, pp. 120-122. doi:10.1016/S0967-5868(02)00269-2

[7] S. Sahaiu-Srivastava and B. Jones, "Brainstem Tuberculoma in the Immunocompetent: Case Report and Literature Review," Clinical Neurology and Neurosurgery, Vol. 110, No. 3, 2008, pp. 302-304. doi:10.1016/j.clineuro.2007.11.002

[8] R. Kumar, R. Jain, A. Kaur and D. K. Chhabra, "Brain Stem Tuberculosis in Children," British Journal of Neurosurgery, Vol. 14, No. 4, 2000, pp. 356-361. doi:10.1080/026886900417397

[9] H. Sugimori, Y. Saku, S. Ibayashi, T. Ogasawara, M. Fujishima and M. Iida, "Solitary Pontine Tuberculoma," Internal Medicine, Vol. 41, No. 9, 2002, pp. 738-742. doi:10.2169/internalmedicine.41.738

[10] Y. Q. Feng, N. Guo, F. Huang, X. Chen, Q. S. Sun and J. X. Liu, “Anti-Tuberculosis Treatment for Devic's Neuromyelitis Optica,” Journal of Clinical Neuroscience, Vol. 17, No. 11, 2010, pp. 1372-1377. doi:10.1016/j.jocn.2010.02.023

[11] Y. Q. Feng, "Infratentorial Tuberculoma Mimics Tumor in Immunocompetent Adults: An Analysis of $11 \mathrm{~Pa}-$ tients,” Journal of Cancer Science \& Therapy, 2012.

[12] A. Unal and P. N. Sutlas, "Clinical and Radiological Features of Symptomatic Central Nervous System Tubercu- lomas,” European Journal of Neurology, Vol. 12, No. 10, 2005, pp. 797-804. doi:10.1111/j.1468-1331.2005.01067.x

[13] G. Sonmez, E. Ozturk, H. O. Sildiroglu, H. Mutlu, F. Cuce, M. G. Senol, A. Kutlu, C. C. Basekim and E. M. R. I. Kizilkaya, "Findings of Intracranial Tuberculomas," Clinical Imaging, Vol. 32, No. 2, 2008, pp. 88-92. doi:10.1016/j.clinimag.2007.08.024

[14] K. Mazodier, E. Bernit, V. Faure, et al., "Central Nervous Tuberculosis in Patients Non-HIV: Seven Case Reports," La Revue de Médecine Interne, Vol. 24, No. 2, 2003, pp. 78-85. doi:10.1016/S0248-8663(02)00715-4

[15] C. Bayindir, O. Mete and B. Bilgic, "Retrospective Study of 23 Pathologically Proven Cases of Central Nervous System Tuberculomas,” Clinical Neurology and Neurosurgery, Vol. 108, No. 4, 2006, pp. 353-357. doi:10.1016/j.clineuro.2005.03.001

[16] A. Bouchama, M. Z. Al-Kawi, I. Kanaan, R. Coates, A. Jallu, B. Rahm and E. B. Siqueira, "Brain Biopsy in Tuberculoma: The Risks and Benefits,” Neurosurgery, Vol. 28, No. 3, 1991, pp. 405-409. doi:10.1227/00006123-199103000-00011

[17] O. Talamás, O. H. Del Brutto and G. García-Ramos, "Brain-Stem Tuberculoma. An Analysis of 11 Patients," Archives of Neurology, Vol. 46, No. 5, 1989, pp. 529-535. doi:10.1001/archneur.1989.00520410063025

[18] H. U. Qureshi, S. N. Merwat, S. A. Nawaz, A. A. Rana, A. Malik, M. K. Mahmud, A. Latif, A. Khan and A. R. Sarwari, "Predictors of Inpatient Mortality in 190 Adult Patients with Tuberculous Meningitis," Journal of Pakistan Medical Association, Vol. 52, No. 4, 2002, pp. 159-163.

[19] S. Haldar, N. Sharma, V. K. Gupta and J. S. Tyagi, "Efficient Diagnosis of Tuberculous Meningitis by Detection of Mycobacterium Tuberculosis DNA in Cerebrospinal Fluid Filtrates Using PCR,” Journal of Medical Microbiology, Vol. 58, No. 5, 2009, pp. 616-624. doi:10.1099/jmm.0.006015-0

[20] Y. Feng, N. Guo, J. Liu, X. Chen, Q. Sun, R. Lai and F. Huang, "Mycobacteria Infection in Incomplete Transverse Myelitis Is Refractory to Steroids: A Pilot Study," Clinical and Developmental Immunology, Vol. 2011, 2011, Article ID: 501369. doi:10.1155/2011/501369

[21] V. C. Cheng, P. L. Ho, R. A. Lee, et al., "Clinical Spectrum of Paradoxical Deterioration during Antituberculosis Therapy in Non-HIV-Infected Patients," European Journal of Clinical Microbiology \& Infectious Diseases, Vol. 21, No. 11, 2002, pp. 803-809. doi:10.1007/s10096-002-0821-2

[22] V. Asselman, F. Thienemann, D. J. Pepper, A. Boulle, R. J. Wilkinson, G. Meintjes and S. Marais, "Central Nervous System Disorders after Starting Antiretroviral Therapy in South Africa,” AIDS, Vol. 24, No. 18, 2010, pp. 2871-2876. doi:10.1097/QAD.0b013e328340fe76 